Creation of stable molecular junctions with a custom-designed scanning tunneling microscope

This article has been downloaded from IOPscience. Please scroll down to see the full text article.

2011 Nanotechnology 22485703

(http://iopscience.iop.org/0957-4484/22/48/485703)

View the table of contents for this issue, or go to the journal homepage for more

Download details:

IP Address: 141.211.173.82

The article was downloaded on 06/04/2012 at 15:57

Please note that terms and conditions apply. 


\title{
Creation of stable molecular junctions with a custom-designed scanning tunneling microscope
}

\author{
Woochul Lee ${ }^{1}$ and Pramod Reddy ${ }^{1,2}$ \\ ${ }^{1}$ Department of Mechanical Engineering, University of Michigan, Ann Arbor, MI 48109, \\ USA \\ ${ }^{2}$ Department of Materials Science, University of Michigan, Ann Arbor, MI 48109, USA \\ E-mail: pramodr@umich.edu
}

Received 26 June 2011, in final form 21 September 2011

Published 9 November 2011

Online at stacks.iop.org/Nano/22/485703

\begin{abstract}
The scanning tunneling microscope break junction (STMBJ) technique is a powerful approach for creating single-molecule junctions and studying electrical transport in them. However, junctions created using the STMBJ technique are usually mechanically stable for relatively short times $(<1 \mathrm{~s})$, impeding detailed studies of their charge transport characteristics. Here, we report a custom-designed scanning tunneling microscope that enables the creation of metal-single molecule-metal junctions that are mechanically stable for more than 1 minute at room temperature. This stability is achieved by a design that minimizes thermal drift as well as the effect of environmental perturbations. The utility of this instrument is demonstrated by performing transition voltage spectroscopy—at the single-molecule level—on

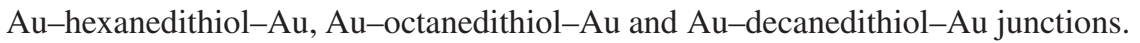

S. Online supplementary data available from stacks.iop.org/Nano/22/485703/mmedia

(Some figures may appear in colour only in the online journal)

\section{Introduction}

Nanoscale molecular junctions provide a unique opportunity to probe quantum transport [1] and are being actively studied due to their potential technological importance in molecular electronics [2] and organic-based thermoelectrics [3-5]. In fact, a variety of interesting effects including negative differential resistance, rectification, switching and gating have all been observed [6] in molecular junctions. Further, recent computational studies [7, 8] have shown that it may be possible to create molecular junctions with large thermoelectric efficiencies using appropriately tailored organic molecules.

A variety of experimental techniques have been developed recently to study charge transport in single-molecule junctions. Some of the widely used techniques include the electromigrated break junction (EBJ) technique [9], the mechanically controllable break junction (MCBJ) technique [10] and the scanning tunneling microscope break junction (STMBJ) technique [11-14]. These techniques often complement each other and are uniquely suited for specific studies. The MCBJ and
STMBJ techniques are closely related and enable trapping single molecules by mechanically manipulating electrodes [6]. The MCBJ technique is well known for its superb mechanical stability and has been widely used in a variety of twoterminal studies of electrical transport in single-molecule junctions $[15,16]$. The STMBJ technique allows for not only two terminal measurements of electrical transport properties but also thermoelectric properties. Further, since the STMBJ technique employs a scanning tunneling microscope [17] it is possible to image the molecules that are being studied, thus enabling more detailed insights [18]. The EBJ technique is well suited for performing three-terminal measurements where the electronic structure of the molecular junction is controlled via a gate electrode. However, the EBJ technique involves a substantial effort in micro-fabricating the break junction devices, impeding the study of a large number of molecular junctions in a short time. In contrast to the EBJ technique, the MCBJ and STMBJ techniques provide a convenient approach for performing a large number of 
two-terminal measurements $(>1000)$ in a short time $(<1 \mathrm{~h})$ enabling a statistical interpretation of results [11].

Recently, the STMBJ technique has been used extensively to study the relationship between the molecular structure and the low bias electrical conductance of molecular junctions [11-14]. However, the STMBJ technique lacks mechanical stability, resulting in single-molecule junctions that are stable only for short periods of time $(<1 \mathrm{~s})$. This poor mechanical stability limits a detailed study of the currentvoltage characteristics and the thermoelectric properties of molecular junctions. This apparent limitation of the STMBJ technique is due to the mechanical drift in the scanning tunneling microscope (STM), which arises from temperature variations and mechanical vibrations.

In this work, we present a custom-designed scanning tunneling microscope (STM) that operates at room temperature in a high vacuum environment $\left(<10^{-6}\right.$ Torr) and is capable of stably trapping a molecular junction for more than $1 \mathrm{~min}-$ at least an order of magnitude better than what was reported in previous studies using the STMBJ technique [13]. This improvement in stability is achieved by incorporating four important features into the instrument: (1) a scanner that is designed to suppress drift associated with temperature changes, (2) a temperature controller that allows excellent temperature stability $( \pm 1 \mathrm{mK})$, (3) vibration isolation to attenuate the effect of ground disturbances and (4) operation in a high vacuum environment to eliminate the effect of acoustic disturbances. We demonstrate that these improvements are sufficient to create single-molecule junctions that are stable for long periods ( $>1 \mathrm{~min}$ ). The utility of this instrument is illustrated by performing transition voltage spectroscopy (TVS) [19] on single-molecule junctions, formed by trapping hexanedithiol (HDT), octanedithiol (ODT) and decanedithiol (DDT) molecules between gold (Au) electrodes to obtain, approximately, the energetic separation between the Fermi level and the closest molecular orbital of the junctions. We believe that this novel instrument will facilitate the study of thermoelectric and thermal transport phenomena in molecular junctions, where it is necessary to perform measurements for long time periods to improve the signal-to-noise ratio.

\section{STM design}

The STM comprises of a scanner, a temperature-controlled radiation shield, control electronics and a vacuum chamber. Among these, the scanner, the temperature-controlled radiation shield and some of the control electronics were custom-built and are described below.

\subsection{STM scanner}

A detailed schematic of the Besocke scanner [20, 21] integrated into the STM is shown in figure 1. The Besocke scanner features four piezoelectric tubes, three of which support a sample plate. A sharp Au tip is mounted on the fourth piezoelectric tube (figure 1), which is at an equidistant position from the other three piezoelectric tubes and is used to scan the sample as well as to trap molecules (explained in detail
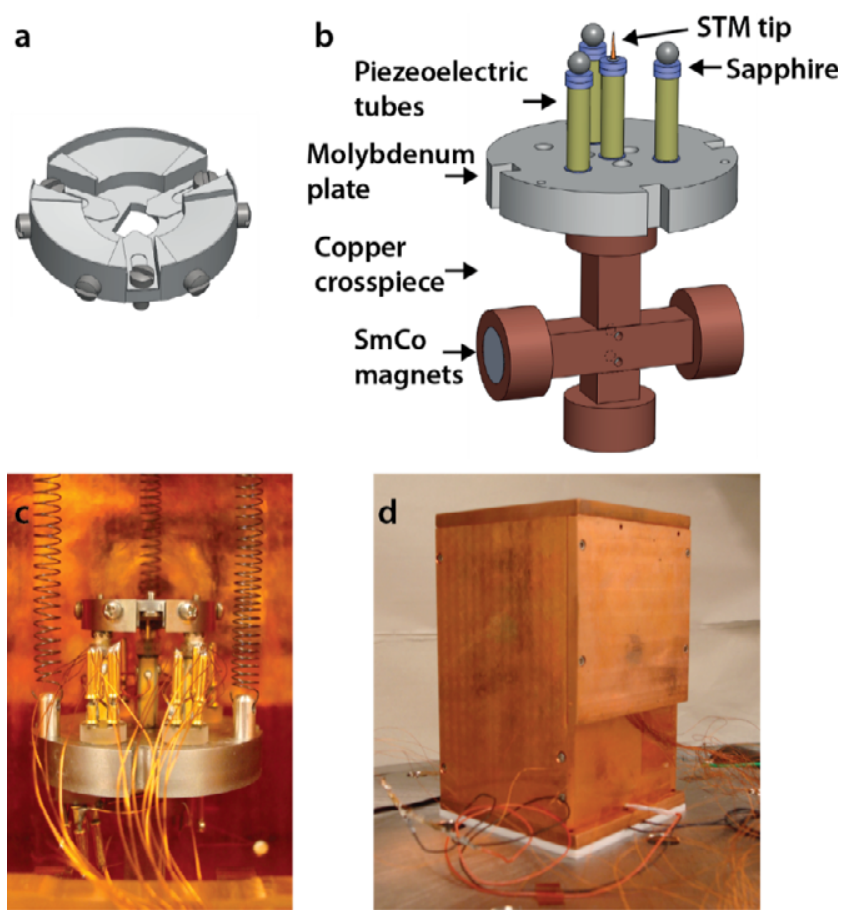

Figure 1. STM scanner assembly and radiation shield. (a) The schematic of the sample plate. (b) The schematic of the Besocke scanner featuring four piezoelectric tubes. (c) The assembly of the sample plate and the STM scanner. (d) The copper enclosure (radiation shield) which houses the scanner.

later). The Besocke scanner is chosen in the present work due to its excellent ability to suppress thermal drift. In general, temperature changes of the entire scanner lead to a thermal expansion/contraction of the piezoelectric tubes. In a Besocke scanner, this expansion/contraction does not translate into large changes in the spacing between the STM tip and the sample plate. This is because the thermal expansion/contraction of the outer piezoelectric tubes supporting the sample plate is almost identical to that of the expansion/contraction of the inner piezoelectric tube resulting in suppression of drift between the tip and the sample. More details regarding the estimated drift of the scanner, scanner fabrication and vibration isolation are provided in the supporting information (SI available at stacks. iop.org/Nano/22/485703/mmedia).

\subsection{Temperature-controlled radiation shield}

The Besocke scanner design described above allows for good thermal compensation, ensuring that the scanner is relatively immune to thermal drift. However, the scanner is not completely unaffected by temperature variations. This is primarily due to the mismatch in the thermal expansion coefficient of the STM tip (made of gold) and that of the tungsten balls mounted on the outer piezoelectric tubes (figure 1(b)). This mismatch results in a drift in the gap between the sample plate and the tip (details in SI available at stacks.iop.org/Nano/22/485703/mmedia) when the temperature changes with time. Therefore, it is essential to ensure that the temperature variations are very small. 
Although the scanner is located in a vacuum environment, it is still thermally coupled to the ambient environment where the temperature varies significantly, $\sim 2$ K over a period of $24 \mathrm{~h}$. This thermal coupling exists because heat can be transported from the ambient environment via the springs and electrical wires that are connected to the scanner (figure 1(c)) and via thermal radiation. In order to minimize the thermal coupling with the ambient, the scanner is placed in a copper enclosure (figure 1(d)), which acts not only as a thermal reservoir but also as a radiation shield. Further, the temperature of the enclosure is maintained at a constant value by a custom-built controller (proportional and integral control). The control system senses temperature changes via a thermistor (Omega, 44033-2252 $\Omega$ ) and attenuates them by modulating the electrical current supplied to an electrical heater integrated into the copper enclosure. Using this approach, it was possible to maintain the temperature of the copper enclosure at a fixed temperature ( $\sim 5 \mathrm{~K}$ above the average ambient temperature) with variations less than $\pm 1 \mathrm{mK}$ in a $3 \mathrm{~h}$ period (details in SI available at stacks.iop.org/Nano/22/485703/mmedia).

\subsection{Scanner control and calibration}

The motion of the STM scanner is controlled by a combination of a customized real-time controller and a commercial STM controller (RHK Technology). The commercial STM controller is used for the coarse and fine approach steps that are essential to place the STM tip in close proximity to the sample surface (i.e. the tunneling region). Further, the commercial STM controller is also used for scanning the surface to obtain topographical information. The realtime controller is implemented using hardware from National Instruments (PXI8110) and is used to increase or decrease the separation between the STM tip and the sample in small increments (as small as $3 \mathrm{pm} / \mathrm{step}$ ). Such control is essential for the creation of stable molecular junctions and is discussed in detail below. In order to calibrate the piezoelectric tubes of the Besocke scanner, we adopted an approach similar to that used by others in the past $[22,23]$. Briefly, the $X-Y$ scan parameters of the piezoelectric tubes were adjusted such that the correct topography of a highly oriented pyrolytic graphite (HOPG) sample was observed [22]. Further, the sensitivity of the piezoelectric tube in the $Z$ direction (perpendicular to the sample) was calibrated by measuring the known step height of an $\mathrm{Au}(111)$ terrace [23].

\section{Single-molecule experiments}

The stability and use of this STM is demonstrated by studying electron transport in single-molecule junctions created from hexanedithiol (HDT), octanedithiol (ODT) and decanedithiol (DDT) molecules. The protocol used to create the Aualkanedithiol-Au junctions is described first. Subsequently, the statistical analysis used for determining the conductance of a single-molecule junction is presented. Finally, we present results that demonstrate that single-molecule junctions created using this STM are mechanically stable for extended periods $(>1 \mathrm{~min})$.

\subsection{Creation of single-molecule junctions}

The first step in the formation of $\mathrm{Au}$-alkanedithiol-Au junctions is to create a monolayer of alkanedithiol molecules on a flat Au surface. The flat Au surface is created using a template stripping technique [24]. In this technique, $\sim 100 \mathrm{~nm}$ of gold is deposited on a pristine silicon wafer. Subsequently, epoxy (Epotek 377) is applied evenly on the gold-coated silicon wafer and a square glass piece $(7 \mathrm{~mm} \times 7 \mathrm{~mm})$ is placed on top of the epoxy. Next, the entire wafer is cured in an oven at $\sim 150{ }^{\circ} \mathrm{C}$ for $\sim 1.5 \mathrm{~h}$. Later, the glass piece is cleaved off from the silicon wafer, resulting in a very smooth $\mathrm{Au}$ film (RMS value $<1 \mathrm{~nm}$ over a $1 \mu \mathrm{m}^{2}$ area) that adheres to the glass surface because of the strong bond between gold and epoxy. To coat the alkanedithiol molecules on the flat Au surface, the gold substrate is immersed into an $\sim 1 \mathrm{mM}$ solution of alkanedithiol in ethanol for more than $12 \mathrm{~h}$. During this time, the alkanedithiol molecules chemically bind to the Au surface through a thiol-Au bond [25]. After taking out the alkanedithiol-coated gold substrate, the substrate is rinsed with ethanol to remove molecules that are not chemically bound to the gold surface. This concludes the process of creating a monolayer of molecules on the surface.

To create $\mathrm{Au}$-alkanedithiol-Au junctions, the moleculecoated $\mathrm{Au}$ surface is mounted onto the sample plate (figure 1(a)) of the STM scanner. Subsequently, a coarse approach is performed to place the sample in close proximity to a sharp Au STM tip (figure 2). Once this is accomplished, the STM tip is driven at $\sim 30 \mathrm{~nm} \mathrm{~s}^{-1}$ towards the gold substrate. During this process, a voltage bias $(0.1 \mathrm{~V})$ is applied between the STM tip and the substrate and the current through the STM tip is constantly monitored. When the tunneling current reaches a large predetermined value indicating a very small separation $(<1 \mathrm{~nm})$ between the tip and sample, an additional voltage of $\sim 0.5 \mathrm{~V}$ corresponding to a displacement $\sim 1 \mathrm{~nm}$ is applied to the piezoelectric tube to ensure that the $\mathrm{Au}$ tip crashes into the flat Au surface. This process is known to ensure the formation of a large number of metal (substrate)-moleculemetal (tip) junctions due to the chemical interaction between the thiol end group of the alkane molecules and the $\mathrm{Au}$ tip [13].

When the STM tip is withdrawn (at $\sim 30 \mathrm{~nm} \mathrm{~s}^{-1}$ ) from the substrate, the molecules trapped between the gold electrodes start breaking away until a single molecule is trapped between the electrodes (figure 2 illustrates this process). During the withdrawal process, the current through the molecular junction is measured using a current amplifier and continuously monitored by a data acquisition system that collects data at a sampling frequency of $40 \mathrm{kHz}$. Representative conductance traces obtained in such experiments with octanedithiol molecules are shown in figure 3(a). These conductance traces show characteristic features (steps) that correspond to the mechanical breaking of the molecular junctions [11, 13].

\subsection{Statistical analysis}

In order to obtain statistically meaningful results, $\sim 1000$ experiments were performed for each molecule (HDT/ODT/DDT) 


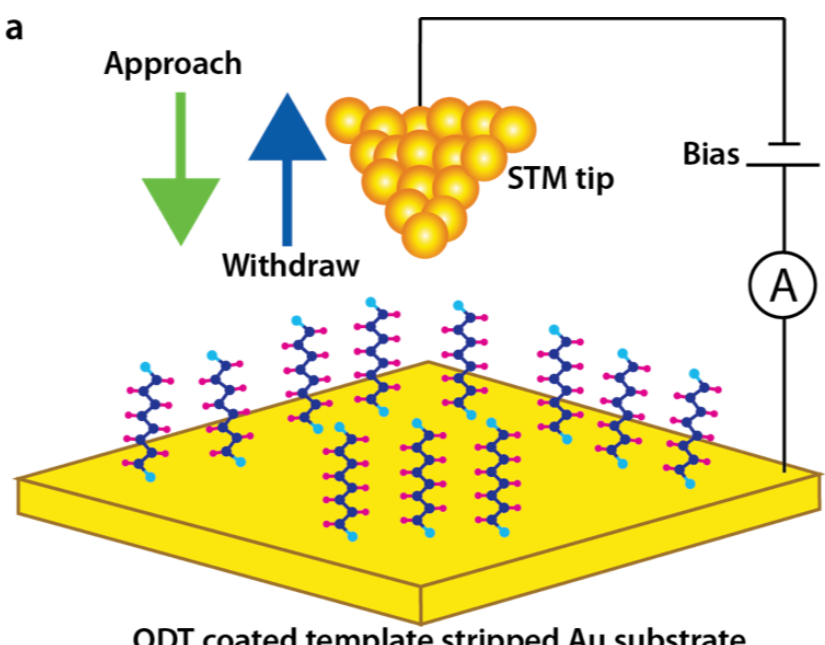

b

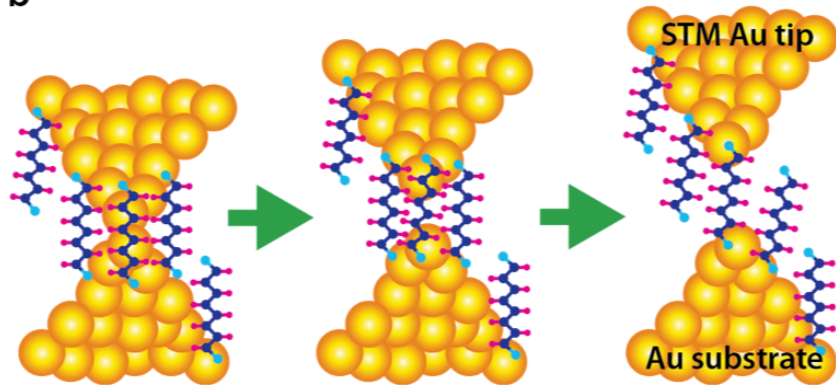

Figure 2. Schematic description of the process of creating single-molecule junctions. (a) An Au STM tip and an octanedithiol (ODT)-coated Au sample are shown. A voltage bias $(+0.1 \mathrm{~V})$ is applied between the STM tip and the substrate and the current through the STM tip is constantly monitored (a positive bias corresponds to a scenario where the substrate is at a higher potential than the STM tip). The STM tip approaches the surface until it makes mechanical contact and is withdrawn immediately after contact. During this process ODT molecules are trapped between the Au electrodes. (b) As the Au STM tip is withdrawn the ODT molecules bridging the two Au electrodes start to break. Ultimately, one molecule bridges the two Au electrodes resulting in a single-molecule junction.

to obtain conductance traces. The conductance traces were analyzed by building a histogram following the approach described by $\mathrm{Xu}$ et al [11]. The conductance histogram obtained from the conductance traces of 1000 junctions of $\mathrm{Au}-$ ODT-Au without any data selection (conventional conductance histogram) is shown in figure 3(b). The conductance histograms of Au-HDT-Au and Au-DDT-Au junctions are shown in the supporting information (available at stacks.iop. org/Nano/22/485703/mmedia). The most probable values of the conductance of an Au-HDT-Au, Au-ODT-Au and AuDDT-Au junction were obtained from a Gaussian fit to the histogram and corresponds to a value of $\sim 2.7 \times 10^{-4} G_{0}$, $\sim 2.2 \times 10^{-5} G_{0}$, and $\sim 5.2 \times 10^{-6} G_{0}$, respectively, where $G_{0}=2 e^{2} / h$ is the quantum of electrical conductance $(e$ is the charge of an electron and $h$ is Planck's constant). Further, the full width at half-maximum (FWHM) of the histograms were found to be $\sim 5.2 \times 10^{-4} G_{0}$ (HDT), $\sim 6.6 \times 10^{-5} G_{0}$ (ODT) and $\sim 1.38 \times 10^{-5} G_{0}$ (DDT) and represent the uncertainty in the measured conductance. In order to verify that the peak

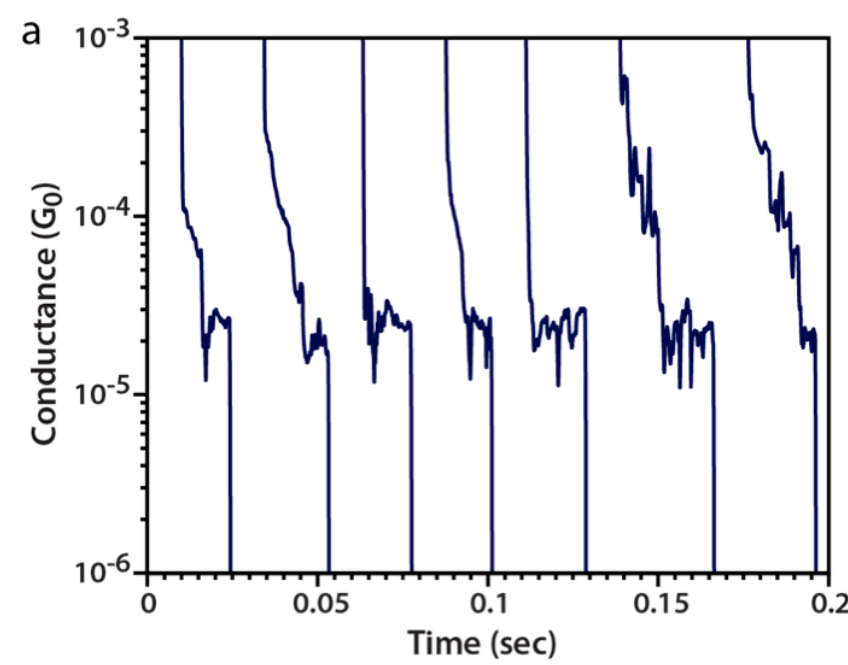

b

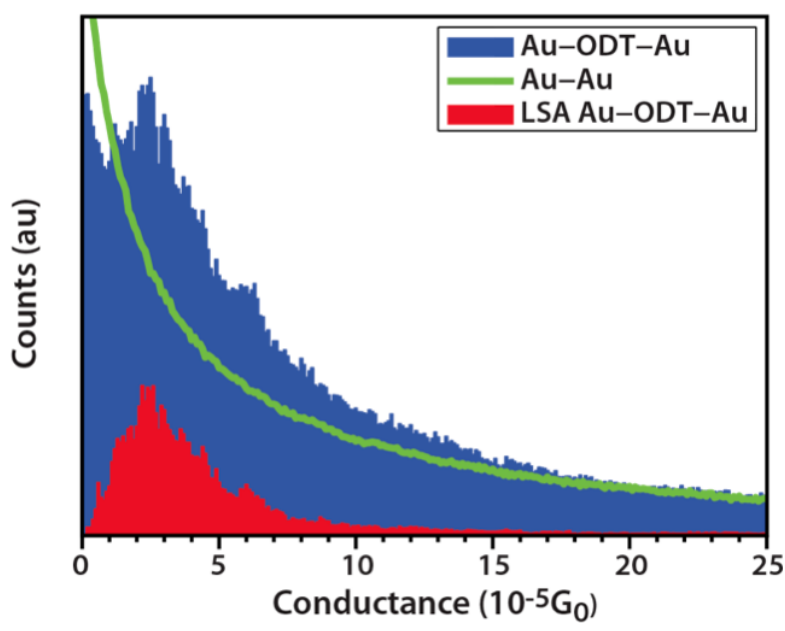

Figure 3. Measurement of the electrical conductance of a single-molecule junction. (a) Conductance traces obtained while withdrawing the STM tip from the sample at $\sim 30 \mathrm{~nm} \mathrm{~s}^{-1}$.

(b) Conductance histogram created from $1000 \mathrm{Au}$-octanedithiol-Au junctions (blue) shows a peak at $\sim 2.2 \times 10^{-5} G_{0}$. Conductance histogram obtained in a control experiment where ODT molecules were not present (green) shows no peak. Conductance histogram obtained from last step analysis (red) shows a peak at $\sim 2.6 \times 10^{-5} G_{0}$.

arises from the alkanedithiol molecules, a control experiment was performed where measurements were performed on a clean Au surface. The histogram (green) obtained in such an experiment is shown in figure 3(b) and does not feature a peak. Similar control experiments were performed for $\mathrm{Au}-$ HDT-Au and Au-DDT-Au junctions and are presented in the supporting information (available at stacks.iop.org/Nano/ 22/485703/mmedia). These experiments clearly confirm that the peak in the histogram is obtained only in the presence of alkanedithiol molecules.

In addition to this conventional histogram-based analysis, we also performed the last step analysis (LSA) [13], which was introduced by one of us, in collaboration with others, in the past. In this analysis, the magnitude of the last step in the conductance traces, which corresponds to the conductance of a single-molecule junction, is measured from a large number $(\sim 1000)$ of conductance traces and is reported in the form 
of a histogram (figure 3(b), and in SI available at stacks.iop. org/Nano/22/485703/mmedia). The histograms have a peak from the Gaussian fit at $\sim 2.8 \times 10^{-4} G_{0}$ (HDT), $\sim 2.6 \times$ $10^{-5} G_{0}$ (ODT) and $\sim 5.5 \times 10^{-6} G_{0}$ (DDT). The corresponding uncertainty of FWHM is $\sim 3.1 \times 10^{-4} G_{0}$ (HDT), $\sim 4.3 \times$ $10^{-5} G_{0}$ (ODT) and $\sim 4 \times 10^{-6} G_{0}$ (DDT). The peak value obtained using LSA corresponds well with the result obtained using the conventional histogram analysis above. In our past work using a commercial STM (molecular imaging, pico-STM) that operated in ambient conditions, it was not possible to obtain a clear peak using conventional histogram analysis without data selection. However, using this new ultrastable STM, a clear peak is also observed in the conventional conductance histogram. We hypothesize that this improvement in the quality results from the superior stability of our STM, which enhances the amount of time that the most probable conductance value of the junction is obtained in a conductance trace.

When the measured electrical resistance of the alkanedithiol junctions is plotted versus the number of carbon atoms in the chain (see SI available at stacks.iop.org/Nano/22/ $485703 /$ mmedia), it is apparent that the electrical resistance increases exponentially with the length of the chain. Such an exponential dependence of the electrical resistance on the length of the molecules is expected because electron transport through Au-alkanedithiol-Au molecular junctions occurs via nonresonant tunneling. Therefore, the resistance of a singlemolecule junctions is proportional to $\exp (\beta N)$, where $N$ is the number of carbon atoms in the alkanedithiol and $\beta$ is the tunneling decay constant. A curve fit (see SI available at stacks. iop.org/Nano/22/485703/mmedia) to the measured electrical resistance of the Au-alkanedithiol-Au junctions suggests that $\beta$ is $\sim 0.99 /$ carbon atom and is in good agreement with previous reports [26].

\subsection{Demonstration of mechanical stability of junctions}

The stability of molecular junctions created using the custombuilt STM was tested at room temperature and in a high vacuum environment. First, single-molecule junctions were created by crashing the STM tip into the substrate as described in section 3.1. Subsequently, the tip was withdrawn from the sample surface at $\sim 0.6 \mathrm{~nm} \mathrm{~s}^{-1}$ until a conductance value below $0.007 G_{0}$ was obtained. After this, the withdrawal speed was further decreased to $\sim 0.003 \mathrm{~nm} \mathrm{~s}^{-1}$. This slow withdrawal process was continued until a conductance plateau was observed around the most probable conductance value as determined from the conductance histograms, suggesting that a single-molecule junction was created. At this time, the $Z$ displacement of the piezoelectric tube was stopped to test for the stability of the molecular junction. A representative plot obtained in one such experiment using Au-ODT-Au junctions is presented in figure 4 and shows that the electrical conductance can be maintained at approximately the same value for $>100$ s. In $\sim 50 \%$ of the experiments performed with HDT, ODT and DDT molecules following this protocol, it was possible to trap junctions for $>100 \mathrm{~s}$. This demonstrates that the custom-designed STM is capable of creating molecular junctions that are stable for more than $1 \mathrm{~min}$.

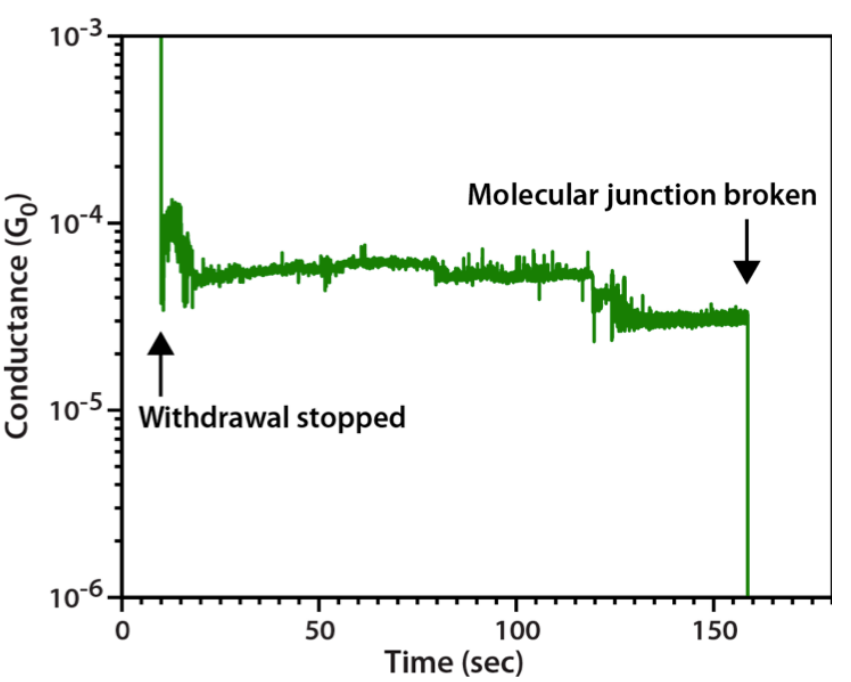

Figure 4. To demonstrate the mechanical stability of molecular junctions the withdrawal of the piezoelectric tube is stopped after the conductance reaches the most probable conductance value (conductance of a single-molecule junction). Subsequently, the conductance of the molecular junction is monitored until the junction breaks down. A representative trace obtained in measurements of $\mathrm{Au}-$ octanedithiol-Au junctions is shown above. The $\mathrm{Au}$-octanedithiol-Au junction is found to be stable for more than 2 min, after which the conductance drops rapidly, indicating a breakdown of the junction.

It can be seen in figure 4 that after about 2 min the conductance of the ODT junction drops rapidly due to the breakdown of the junction. Understanding the mechanism for the breakdown of the molecular junctions after $\sim 1-$ $2 \mathrm{~min}$ is critical in exploring the possibility of further improving the stability of junctions. Does the junction break due to mechanical drift or due to thermally driven breakdown of the Au-thiol or $\mathrm{Au}-\mathrm{Au}$ bonds? We believe that the small thermal drift of the STM scanner ultimately results in increasing the separation between the STM tip and the substrate, leading to a mechanical breakdown of the Au-ODT-Au junction. This hypothesis is supported by previous experimental studies [27] where the thermodynamic stability of $\mathrm{Au}-$ molecule-Au junctions created from thiolterminated molecules was studied at room temperature using a mechanically controllable break junction technique. These studies [27] suggest that the lifetime molecular junctions, created by trapping thiol-terminated molecules between gold electrodes, should be $>1 \mathrm{~h}$ if thermal drift is attenuated further. Therefore, it should be possible to further improve the stability of molecular junctions by attenuating the temperature variations below the current level $( \pm 1 \mathrm{mK})$.

\section{Transition voltage spectroscopy of single-molecule junctions}

A new experimental technique-transition voltage spectroscopy (TVS) - that can determine, approximately, the energetic separation between the Fermi level and closest molecular orbital of the junctions has been established 
recently [28, 19, 29]. In TVS the current-voltage characteristics of molecular junctions are analyzed by plotting a Fowler-Nordheim $(\mathrm{FN})$ curve, $\ln \left(I / V^{2}\right)$ against $(1 / V)$. For asymmetric molecular junctions, an FN plot is expected to feature a minimum value for either positive or negative bias voltages [30]. However, if the junction is symmetric the FN plot would feature two minima (one each for positive and negative biases) that have almost the same magnitude [30]. Recently, TVS has been interpreted within a coherent electron transport model (Landauer formalism) by Huisman et al and Araidai et al [31, 29]. In this approach, the transmission function of the molecular junction is computed and typically features peaks at energies corresponding to the HOMO and LUMO orbitals of the molecular junction. Within this model it has been suggested that the minima in the FN plot occurs when a certain fraction of the transmission function enters the bias window between the quasi-Fermi levels of the electrodes. The minima obtained using Fowler-Nordheim curves is called the transition voltage ( $V_{\text {trans }}$ ) and is expected to approximately indicate the energetic separation $\left(\Delta \sim\left|e V_{\text {trans }}\right|\right)$ between the closest molecular orbital and the chemical potential (Fermi level).

Recently, several researchers have used transition voltage spectroscopy to qualitatively study the effect of molecular structure, gate voltage and molecular length on the energetic separation of molecular junctions [28, 32, 33]. However, most of these studies were performed either in junctions where multiple molecules were trapped between electrodes or in single-molecule junctions created using the electromigrated break junction technique at low temperatures $(4.2 \mathrm{~K})[32,34]$. Here we demonstrate the utility of the ultra-stable STM by performing TVS—in single-molecule junctions_-at room temperature.

In order to obtain the $I-V$ characteristics of singlemolecule junctions, which are necessary for performing TVS, the withdrawal process (described in section 3.3) is stopped when the conductance equals that of a singlemolecule junction. Subsequently, the voltage bias is varied linearly between -2 and $+2 \mathrm{~V}$ while monitoring the electric current flowing through the junction. We note that, in our experiments, a positive bias corresponds to a scenario where the substrate is at a higher potential than the STM tip. Figure 5(a) shows a conductance trace for ODT reflecting the procedure described above. The region highlighted in green (figure 5(a)) represents the location where the voltage sweep is performed. Two representative $I-V$ characteristics obtained in such experiments are shown in the inset of figure 5(b). The corresponding FN plots obtained using these $I-V$ curves are shown in figure 5(b). The FN plot (red circles) features a minima on both sides and corresponds to the more symmetric $I-V$ curve (solid red line in the inset of figure 5(b)). The two minima are at $-0.697 \mathrm{~V}^{-1}$ and $0.6810 \mathrm{~V}^{-1}$ and correspond to transition voltages of $-1.43 \mathrm{~V}$ and $+1.47 \mathrm{~V}$, respectively, suggesting an apparent energetic separation of $\sim 1.45 \mathrm{eV}$ (between the Fermi level and the closest molecular orbital) for $\mathrm{Au}-\mathrm{ODT}-\mathrm{Au}$ junctions. The FN plot (blue squares) features only one minima at $\sim-0.63 \mathrm{~V}^{-1}$ and corresponds to the less symmetric $I-V$ curve (dashed blue line in the inset of
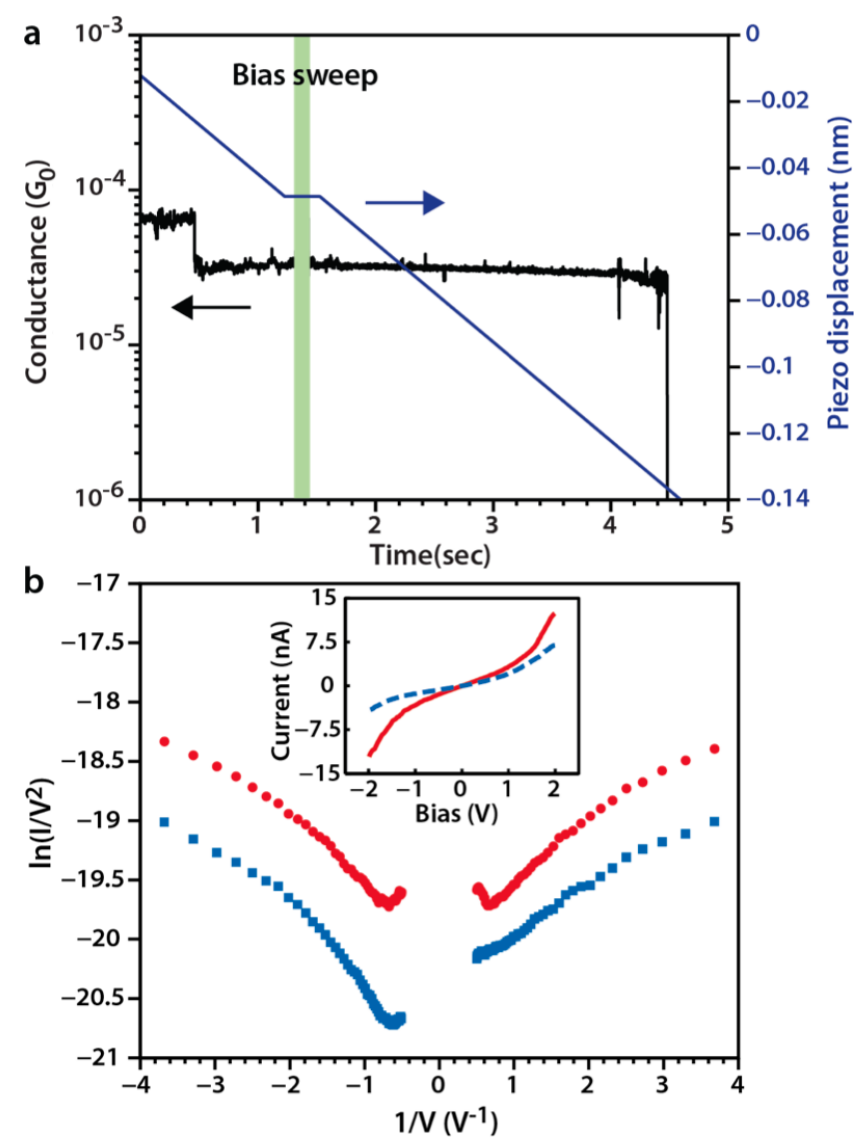

Figure 5. Current-voltage $(I-V)$ characteristics and

Fowler-Nordheim plots of Au-ODT-Au junctions. (a) The Au STM tip is withdrawn until an electrical conductance of $\sim 2.6 \times 10^{-5} G_{0}$ (the most probable conductance value) is obtained after which the withdrawal process is stopped (the region shown in green) and the voltage is varied from -2 to $+2 \mathrm{~V}$ to obtain the $I-V$ characteristics The conductance value remains the same after sweeping the voltage bias, indicating that the molecular junction is not destroyed by the large bias. (b) The inset shows two representative $I-V$ curves obtained in measurements. The $I-V$ curve shown in red (solid line) is symmetric and has a Fowler-Nordheim plot (red circles) that features minima at $-1.43 \mathrm{~V}$ and $+1.47 \mathrm{~V}$, respectively. The $I-V$ curve shown in blue (dashed line) is asymmetric and has a

Fowler-Nordheim plot (blue squares) that features only one minima at $-1.6 \mathrm{~V}$.

figure 5(b)). We note that for HDT, ODT and DDT junctions, among the $300 I-V$ curves that were obtained for each junction only $\sim 50$ showed a minimum for both negative and positive voltages. Based on past theoretical work [30, 35], we believe that the $I-V$ characteristics that show both a positive and negative $V_{\text {trans }}$, with an almost identical magnitude, correspond to molecular junctions that are symmetric in their geometry.

In order to obtain statistically meaningful results, positive and negative transition voltages were obtained in $\sim 50$ independent experiments each for $\mathrm{Au}-\mathrm{HDT}-\mathrm{Au}, \mathrm{Au}-\mathrm{ODT}-\mathrm{Au}$ and $\mathrm{Au}-\mathrm{DDT}-\mathrm{Au}$ junctions. The mean value and standard deviation of the obtained transition voltages are presented in table 1. This data suggests that for each molecular junction the measured positive and negative transition voltages are similar in magnitude, as expected for a geometrically symmetric molecular junction [30]. Further, the relative invariance of 
Table 1. To obtain statistically meaningful results, positive and negative transition voltages were obtained in $\sim 50$ independent single-molecule experiments each for Au-HDT-Au, Au-ODT-Au and $\mathrm{Au}-\mathrm{DDT}-\mathrm{Au}$ junctions. The mean value and standard deviation of the measured transition voltages are shown.

\begin{tabular}{lrl}
\hline & $+V_{\text {trans }}$ & \multicolumn{1}{l}{$-V_{\text {trans }}$} \\
\hline Au-HDT-Au & $1.39 \pm 0.21$ & $-1.37 \pm 0.23$ \\
Au-ODT-Au & $1.35 \pm 0.14$ & $-1.40 \pm 0.17$ \\
Au-DDT-Au & $1.37 \pm 0.18$ & $-1.44 \pm 0.17$ \\
\hline
\end{tabular}

the magnitude of the positive and negative transition voltages when the length of the molecular junctions is varied indicates that the energetic separation between the chemical potential and the closest molecular orbital is approximately constant in alkanedithiol junctions. This result is consistent with previous studies [28] on alkanemonothiol junctions, where a similar length independence was observed.

\section{Discussion and conclusion}

We described a custom-built STM, which enables the formation of molecular junctions that are mechanically stable for time periods as long as $2 \mathrm{~min}$ at room temperature. This represents at least an order-of-magnitude improvement over the stability observed in past STMBJ measurements. This improvement is accomplished by incorporating a Besocke scanner, which is relatively immune to temperature variations, by active control of the temperature, and by attenuating the effect of acoustic and ground vibrations. We demonstrated the utility of this stable instrument by performing transition voltage spectroscopy at the single-molecule level. We believe that this improved stability will not only enable more detailed electrical transport studies such as transition voltage spectroscopy at the single-molecule level but will also facilitate the study of thermoelectric and thermal transport phenomena [37] in molecular junctions, where it is necessary to perform measurements for long time periods to improve the signal-to-noise ratio. In fact, one of the major goals of our future work is to integrate this ultra-stable STM with a picowatt-resolution calorimeter [36] to probe heat transport in molecular junctions with picowatt resolution.

\section{Acknowledgments}

PR gratefully acknowledges support from the NSF through an NSF-CAREER award (CBET 0844902). WL thanks Wonho Jeong for help in building the STM scanner. WL and PR thank Edgar Meyhofer for valuable discussions.

\section{References}

[1] Datta S 2005 Quantum Transport: Atom to Transistor (Cambridge: Cambridge University Press)

[2] Nitzan A and Ratner M A 2003 Electron transport in molecular wire junctions Science 300 1384-9

[3] Reddy P, Jang S Y, Segalman R A and Majumdar A 2007 Thermoelectricity in molecular junctions Science 315 1568-71
[4] Malen J A, Yee S K, Majumdar A and Segalman R A 2010 Fundamentals of energy transport, energy conversion, and thermal properties in organic-inorganic heterojunctions Chem. Phys. Lett. 491 109-22

[5] Tan A, Balachandran J, Sadat S, Gavini V, Dunietz B D, Jang S Y and Reddy P 2011 Effect of length and contact chemistry on the electronic structure and thermoelectric properties of molecular junctions J. Am. Chem. Soc. $1338838-41$

[6] Tao N J 2006 Electron transport in molecular junctions Nature Nanotechnol. 1 173-81

[7] Bergfield J P, Solis M A and Stafford C A 2010 Giant thermoelectric effect from transmission supernodes ACS Nano 4 5314-20

[8] Finch C M, Garcia-Suarez V M and Lambert C J 2009 Giant thermopower and figure of merit in single-molecule devices Phys. Rev. B 79033405

[9] Park H, Park J, Lim A, Anderson E, Alivisatos A and McEuen P 2000 Nanomechanical oscillations in a single-C-60 transistor Nature 407 57-60

[10] Tsutsui M, Taniguchi M and Kawai T 2008 Local heating in metal-molecule-metal junctions Nano Lett. 8 3293-7

[11] Xu B and Tao N 2003 Measurement of single-molecule resistance by repeated formation of molecular junctions Science 301 1221-3

[12] Venkataraman L, Klare J, Nuckolls C, Hybertsen M and Steigerwald M 2006 Dependence of single-molecule junction conductance on molecular conformation Nature 442 904-7

[13] Jang S Y, Reddy P, Majumdar A and Segalman R A 2006 Interpretation of Stochastic events in single molecule conductance measurements Nano Lett. 6 2362-7

[14] Mishchenko A et al 2010 Influence of conformation on conductance of biphenyl-dithiol single-molecule contacts Nano Lett. 10 156-63

[15] van Ruitenbeek J M, Smit R H M, Noat Y, Untiedt C, Lang N D and van Hemert M C 2002 Measurement of the conductance of a hydrogen molecule Nature 419 906-9

[16] Dulić D, Pump F, Campidelli S, Lavie P, Cuniberti G and Filoramo A 2009 Controlled stability of molecular junctions Angew. Chem. Int. Ed. 48 8273-6

[17] Chen C J 2008 Introduction to Scanning Runneling Microscopy (Oxford: Oxford University Press)

[18] Lafferentz L, Ample F, Yu H, Hecht S, Joachim C and Grill L 2009 Conductance of a single conjugated polymer as a continuous function of its length Science 323 1193-7

[19] Beebe J M, Kim B, Gadzuk J W, Frisbie C D and Kushmerick J G 2006 Transition from direct tunneling to field emission in metal-molecule-metal junctions Phys. Rev. Lett. 97026801

[20] Besocke K 1987 An easily operable scanning tunneling microscope Surf. Sci. 181 145-53

[21] Stipe B C, Rezaei M A and Ho W 1999 A variable-temperature scanning tunneling microscope capable of single-molecule vibrational spectroscopy Rev. Sci. Instrum. 70 137-43

[22] Osing J and Shvets I V 1998 Bulk defects in graphite observed with a scanning tunnelling microscope Surf. Sci. 417 145-50

[23] Hallmark V M, Chiang S, Rabolt J F, Swalen J D and Wilson R J 1987 Observation of atomic corrugation on $\mathrm{Au}(111)$ by scanning tunneling microscopy Phys. Rev. Lett. 592879

[24] Hegner M, Wagner P and Semenza G 1993 Ultralarge atomically flat template-stripped Au surfaces for scanning probe microscopy Surf. Sci. 291 39-46

[25] Love J C, Estroff L A, Kriebel J K, Nuzzo R G and Whitesides G M 2005 Self-assembled monolayers of thiolates on metals as a form of nanotechnology Chem. Rev. $1051103-69$

[26] Engelkes V B, Beebe J M and Frisbie C D 2004 Length-dependent transport in molecular junctions based on SAMs of alkanethiols and alkanedithiols: effect of metal work function and applied bias on tunneling efficiency and contact resistance J. Am. Chem. Soc. 126 14287-96 
[27] Tsutsui M, Shoji K, Morimoto K, Taniguchi M and Kawai T 2008 Thermodynamic stability of single molecule junctions Appl. Phys. Lett. 92223110

[28] Beebe J M, Kim B, Frisbie C D and Kushmerick J G 2008 Measuring relative barrier heights in molecular electronic junctions with transition voltage spectroscopy ACS Nano 2 827-32

[29] Huisman E H, Guedon C M, van Wees B J and van der Molen S J 2009 Interpretation of transition voltage spectroscopy Nano Lett. $93909-13$

[30] Mirjani F, Thijssen J M and van der Molen S J 2011 Advantages and limitations of transition voltage spectroscopy: a theoretical analysis Phys. Rev. B 84115402

[31] Araidai M and Tsukada $M$ Theoretical calculations of electron transport in molecular junctions: inflection behavior in Fowler-Nordheim plot and its origin Phys. Rev. B 81235114
[32] Song H, Kim Y, Jang Y H, Jeong H, Reed M A and Lee T 2009 Observation of molecular orbital gating Nature 462 1039-43

[33] Tan A, Sadat S and Reddy P Measurement of thermopower and current-voltage characteristics of molecular junctions to identify orbital alignment Appl. Phys. Lett. 96013110

[34] Song H, Kim Y, Jeong H, Reed M A and Lee T 2010 Coherent tunneling transport in molecular junctions J. Phys. Chem. C $11420431-5$

[35] Chen J Z, Markussen T and Thygesen K S 2010 Quantifying transition voltage spectroscopy of molecular junctions: $a b$ initio calculations Phys. Rev. B 82121412

[36] Sadat S, Chua Y J, Lee W, Ganjeh Y, Kurabayashi K, Meyhofer E and Reddy P 2011 Room temperature picowatt-resolution calorimetry Appl. Phys. Lett. 99043106

[37] Dubi Y and Di Ventra M 2011 Colloquium: Heat flow and thermoelectricity in atomic and molecular junctions Rev Mod. Phys. 83 131-55 